\title{
Novel rotundic acid derivatives: Synthesis, structural characterization and in vitro antitumor activity
}

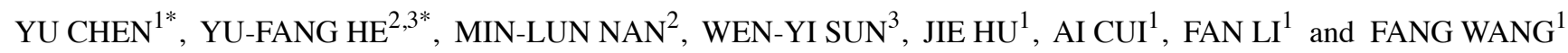 \\ ${ }^{1}$ Department of Pathogenobiology, Norman Bethune College of Medicine, Jilin University, ${ }^{2}$ Jilin Academy of Chinese \\ Medicine Sciences, ${ }^{3}$ School of Pharmaceutical Sciences, Jilin University, Changchun, Jilin 130021, P.R. China
}

Received September 25, 2012; Accepted November 16, 2012

DOI: 10.3892/ijmm.2012.1206

\begin{abstract}
Six novel rotundic acid (RA, 1) derivatives $4 \mathrm{a}-4 \mathrm{f}$ modified at the $28-\mathrm{COOH}$ position were synthesized, and their structures were confirmed by IR, MS, ${ }^{1} \mathrm{H}$ NMR and ${ }^{13} \mathrm{C}$ NMR. The derivatives were evaluated for cytotoxic properties on the following three tumor cell lines: HeLa, HepG2 and SGC-7901. Compound $4 \mathrm{f}$ showed better cytotoxic activity compared with RA treatment and lower $\mathrm{IC}_{50}(4.16 \mu \mathrm{M})$ on HepG2 cells than on HeLa $(8.54 \mu \mathrm{M})$ and SGC-7901 cells $(11.32 \mu \mathrm{M})$. The anticancer mechanism of compound $4 \mathrm{f}$ was studied through cell cycle progression and apoptosis. Notably, compound $4 \mathrm{f}$ was able to induce apoptosis and G0/G1 cell cycle arrest of HepG2 at a concentration of $4.16 \mu \mathrm{M}$. In summary, RA was modified to obtain six novel derivatives. Compound $4 \mathrm{f}$ exhibited better cytotoxicity and may be developed as a potential agent against hepatocellular carcinoma.
\end{abstract}

\section{Introduction}

Hepatocellular carcinoma (HCC) is the fifth-most common malignant tumor in the world (1). The incidence of $\mathrm{HCC}$ has been on the rise in recent years $(1,2)$, and more than $70 \%$ of all newly diagnosed cases of liver cancer occur in Asia (3). The treatment for HCC usually involves surgical resection or liver transplantation with curative options for the patients, when the disease is diagnosed at an early stage (4). However, only $30 \%$ of the patients are eligible for the curative treatment, and recurrence is a common concern affecting up to $70 \%$ of the patients after tumor ablation (5). Only a few non-curative treatment options exist for such patients. Some effective treatment modalities include: ethanol ablation, radiofrequency ablation,

Correspondence to: Dr Fang Wang, Department of Pathogenobiology, Bethune College of Medicine, Jilin University, 126 Xinmin Street, Changchun, Jilin 130021, P.R. China

E-mail:wf@jlu.edu.cn

*Contributed equally

Key words: antitumor activity, apoptosis, characterization, cytotoxicity, derivative, rotundic acid, synthesis transarterial chemoembolization, and selective radiation of lesions (6). Therefore, new therapeutic agents for the treatment of hepatoma need to be explored.

Rotundic acid (RA, 1) (Fig. 1) is one of the pentacyclic triterpenoids, mainly found in Ilex rotunda, Ilex purpurea and other Aquifoliaceae plants (7-10). RA can also be isolated from Mussaenda Pubescens, Olea europaea and Planchonella duclitan (11-13). RA and its derivatives inhibit cell growth. Due to its unstable nature during the metabolic processes, RA induces serious gastrointestinal adverse effects. A considerable number of patents on RA and its derivatives have been applied by our research group in the recent years. Our studies explored these novel compounds extensively and focused on reducing these adverse effects (14-17). In our previous study, eight amino acid derivatives of RA at the $28-\mathrm{COOH}$ position were synthesized, and their in vitro cytotoxic properties were evaluated on three tumor cell lines, A375 (human malignant melanoma cells), HepG2 (human hepatoma cells), and NCI-H446 (human small cell lung cancer cells) (18).

The present study aimed at synthesizing RA derivatives by making structural modifications at the $28-\mathrm{COOH}$ position of RA. The synthesized compounds were characterized and their cytotoxic effects in the three cell lines, HeLa (human cervical cancer), HepG2 (human hepatoma cell) and SGC-7901 (human gastric carcinoma), were studied.

\section{Materials and methods}

Based on the natural structure of RA, six new derivatives $4 \mathrm{a}-4 \mathrm{f}$ were designed and synthesized in this study to improve its bioactivity. Their structures were elucidated on the basis of spectroscopic assays such as IR, MS, ${ }^{1} \mathrm{H}$ NMR and ${ }^{13} \mathrm{C}$ NMR. The cytotoxicity and antitumor effects of RA derivatives were assayed by MTT colorimetric assay with the HeLa, HepG2 and SGC-7901 cell lines. Cell cycle distribution and measurement of the percentage of apoptotic cells were performed by flow cytometry, following staining with propidium iodide (PI) and Annexin V-FITC.

Reagents and cell culture. Reagent-grade chemicals and solvents were obtained from Sigma Chemicals, Munich, Germany. All solvents were freshly distilled and dried prior to use, according to the standard procedures. The HeLa, HepG2 and SGC-7901 cell lines were purchased from the Tumor Center of the Chinese 
Academy of Medical Sciences, Beijing, China. Cells were maintained in Dulbecco's modified Eagle's medium (DMEM) containing $10 \%$ FBS, penicillin $(100 \mathrm{U} / \mathrm{ml})$ and streptomycin $(100 \mu \mathrm{g} / \mathrm{ml})$ at $37^{\circ} \mathrm{C}$ in a humidified atmosphere of $5 \% \mathrm{CO}_{2}$.

Preparation of $R A$. The preparation of RA was reported in our previous study (18). The purity of RA used was $\geq 98 \%$ (HPLC assay) and the extraction yield of RA in our study was up to $100 \mathrm{mg} / \mathrm{g}$, which made it suitable for industry production. Melting points were determined on a Fisher-Johns apparatus and were uncorrected. IR spectra were recorded on a Perkin-Elmer 983G spectrometer. NMR spectra were measured in $\mathrm{C}_{5} \mathrm{D}_{5} \mathrm{~N}$ on a Bruker AM-400 spectrometer, using tetramethylsilane as an internal standard. Coupling constants ( $J$ values) were given in $\mathrm{Hz}$, and an MS Agilent 1100 Series LC/MSD ion-trap mass spectrometer was used to record the HR-ESI-MS.

Structure modification of $R A$ and synthesis of derivatives. The synthetic routes to the RA derivatives are outlined in Fig. 2. Firstly, RA (1) was converted to its 28 -ethyl acetate (2), which was then treated with hydrazine hydrate to give the 28-acetohydrazide (3). It was then reacted with the appropriate aldehyde (benzaldehyde, $\mathrm{p}$-methyl benzaldehyde, $\mathrm{p}$-chloride benzaldehyde, m-nitryl benzaldehyde, p-nitryl benzaldehyde, p-methoxy benzaldehyde) in the presence of glacial acetic acid to give the benzaldehyde $\{2-[(3 \beta, 19 \alpha, 23$-trihydroxy-urs-12en-28-oyl)-hydroxy]-acetic\} hydrazone 4a-4f. The structures of these synthesized compounds were confirmed by IR, MS, ${ }^{1} \mathrm{H}$ NMR and ${ }^{13} \mathrm{C}$ NMR (25-28). All six compounds obtained were synthesized in high yields with purities of $98 \%$ or better and are reported for the first time.

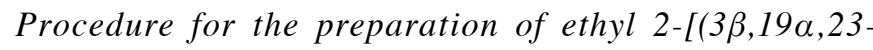
trihydroxy-urs-12-en-28-oyl)-hydroxy]-acetate (2). RA $(10 \mathrm{mmol})$ was dissolved in acetone $(500 \mathrm{ml})$ and heated to clarity. Potassium carbonate $(100 \mathrm{mmol})$, potassium iodide $(0.6 \mathrm{mmol})$, and triethylamine $(6 \mathrm{ml})$ were added to it. Ethyl chloroacetate $(5 \mathrm{ml})$ was then added drop-wise and refluxed for $24 \mathrm{~h}$. The refluxed content was filtered, and the filtrate was concentrated to $100 \mathrm{ml}$. Saturated salt water $(100 \mathrm{ml})$ was added and was extracted with ethyl acetate five times $(30 \mathrm{ml}$ each time). It was then washed with saturated sodium carbonate and water four times ( $25 \mathrm{ml}$ each time). Anhydrous sodium sulfate was added and filtered. The filtrate was evaporated to dryness. The crude was purified by column chromatography on a silica gel column with petroleum ether, ethyl acetate, and formic acid as eluents to give compound 2 as a white powder. The yield was $83.9 \%$, and its melting point was between 133 and $135^{\circ} \mathrm{C}$. IR $(\mathrm{KBr}) \mathrm{cm}^{-1}: 3545,3404,3250,2929,2882,1765$, 1715, 1634, 1469, 1455, 1381, 1207, 1148, 1055 and 1005; ${ }^{1} \mathrm{H}$ NMR $\left(400 \mathrm{MHz}, \mathrm{C}_{5} \mathrm{D}_{5} \mathrm{~N}\right) \delta: 5.40(1 \mathrm{H}, \mathrm{m}, \mathrm{H}-12), 5.06(1 \mathrm{H}$, m, H-3), $4.78\left(2 \mathrm{H}, \mathrm{dd}, J=15.6,27.6 \mathrm{~Hz}, \mathrm{H}-2^{\prime}\right), 4.07(2 \mathrm{H}, \mathrm{q}$, $\left.J=7.2 \mathrm{~Hz}, \mathrm{H}-1^{\prime \prime}\right), 4.06(1 \mathrm{H}, \mathrm{d}, J=10.4 \mathrm{~Hz}, \mathrm{H}-23 \mathrm{a}), 3.61(1 \mathrm{H}, \mathrm{d}$, $J=10.4 \mathrm{~Hz}, \mathrm{H}-23 \mathrm{~b}), 2.75$ (1H, br s, $\mathrm{H}-18), 1.51$ (3H, s, $\left.\mathrm{CH}_{3}-25\right)$, $1.26\left(3 \mathrm{H}, \mathrm{s}, \mathrm{CH}_{3}-27\right), 1.03$ (3H, t, J=7.2 Hz, $\left.\mathrm{CH}_{3}-2 "\right), 0.97$ (3H, d, J=6.64 Hz, $\left.\mathrm{CH}_{3}-30\right), 0.96$ (3H, s, $\left.\mathrm{CH}_{3}-29\right), 0.93$ (3H, s, $\left.\mathrm{CH}_{3}-26\right), 0.82\left(3 \mathrm{H}, \mathrm{s}, \mathrm{CH}_{3}-24\right) ;{ }^{13} \mathrm{C} \mathrm{NMR}\left(100 \mathrm{MHz}, \mathrm{C}_{5} \mathrm{D}_{5} \mathrm{~N}\right) \mathrm{\delta}$ : 177.7 (C-28), 139.4 (C-13), 128.6 (C-12), 73.7 (C-3), 72.7 (C-19), 68.1 (C-23), 54.5 (C-18), 48.8 (C-5), 48.8 (C-9), 47.8 (C-17), 43.0 (C-20), 42.2 (C-14), 42.1 (C-8), 40.5 (C-1), 39.0 (C-4), 38.0

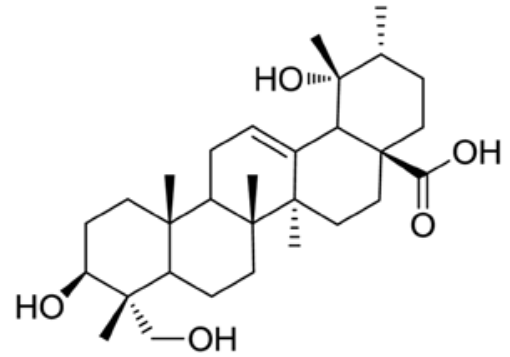

Figure 1. Chemical structure of rotundic acid.

(C-22), 37.3 (C-10), 33.3 (C-7), 29.1 (C-15), 27.8 (C-21), 27.0 (C-29), 26.8 (C-2), 26.3 (C-16), 24.7 (C-27), 24.2 (C-11), 18.8 (C-6), 17.2 (C-25), 16.8 (C-26), 16.2 (C-30), 13.2 (C-24), 61.3 (C-2'), 168.7 (C-1'), 61.0 (C-1"), 14.3 (C-2"). HR-ESI-MS found 575.3953. Calculated 575.3948 for $\mathrm{C}_{34} \mathrm{H}_{55} \mathrm{O}_{7}[(\mathrm{M}+\mathrm{H})+]$.

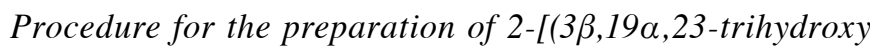
-urs-12-en-28-oyl)-hydroxy]-acetic hydrazide (3). Compound $2(15 \mathrm{mmol})$ was dissolved in absolute ethyl alcohol $(\mathrm{EtOH})(90 \mathrm{ml})$ and $80 \%$ hydrazine hydrate $(7.5 \mathrm{ml})$ was added. The mixture was refluxed for $11 \mathrm{~h}$ and the solvent was removed under reduced pressure using a rotary evaporator to $50 \mathrm{ml}$. Water $(250 \mathrm{ml})$ was added and extracted with chloroform five times ( $50 \mathrm{ml}$ each time). It was then washed with saturated salt water four times ( $50 \mathrm{ml}$ each time). Anhydrous sodium sulfate was added and filtered. The filtrate was evaporated to dryness to yield crude, which was then purified by column chromatography on a silica gel column with petroleum ether and ethyl acetate as eluents to give compound 3 as colorless needles. The yield was $97.7 \%$ and its melting point was between 225 and $226^{\circ} \mathrm{C}$. IR(KBr) $\mathrm{cm}^{-1}: 3624,3398,3335$, 2930, 2876, 1731, 1683, 1450, 1387, 1206, 1147, 1046 and 756; ${ }^{1} \mathrm{H}$ NMR $\left(400 \mathrm{MHz}, \mathrm{C}_{5} \mathrm{D}_{5} \mathrm{~N}\right)$ 8: $9.76(2 \mathrm{H}, \mathrm{s},-\mathrm{NH} 2), 7.45(1 \mathrm{H}$, s, $-\mathrm{NH}), 5.43(1 \mathrm{H}, \mathrm{m}, \mathrm{H}-12), 5.04(1 \mathrm{H}, \mathrm{m}, \mathrm{H}-3), 4.85(2 \mathrm{H}, \mathrm{dd}$, $\left.J=14.2,33.2 \mathrm{~Hz}, \mathrm{H}-1^{\prime}\right), 4.06(1 \mathrm{H}, \mathrm{d}, J=10.4 \mathrm{~Hz}, \mathrm{H}-23 \mathrm{a}), 3.60$ $(1 \mathrm{H}, \mathrm{d}, J=10.4 \mathrm{~Hz}, \mathrm{H}-23 \mathrm{~b}), 2.71(1 \mathrm{H}$, br s, H-18), $1.50(3 \mathrm{H}$, s, $\left.\mathrm{CH}_{3}-25\right), 1.23\left(3 \mathrm{H}, \mathrm{s}, \mathrm{CH}_{3}-27\right), 0.96\left(3 \mathrm{H}, \mathrm{s}, \mathrm{CH}_{3}-29\right), 0.94$ $\left(3 \mathrm{H}, \mathrm{d}, J=6.64 \mathrm{~Hz}, \mathrm{CH}_{3}-30\right), 0.90\left(3 \mathrm{H}, \mathrm{s}, \mathrm{CH}_{3}-26\right), 0.79$ (3H, s, $\left.\mathrm{CH}_{3}-24\right) ;{ }^{13} \mathrm{C}$ NMR $\left(100 \mathrm{MHz}, \mathrm{C}_{5} \mathrm{D}_{5} \mathrm{~N}\right)$ d: $177.5(\mathrm{C}-28), 139.7$ (C-13), 128.5 (C-12), 73.6 (C-3), 72.7 (C-19), 68.1 (C-23), 54.5 (C-18), 48.8 (C-5), 48.7 (C-9), 47.8 (C-17), 43.0 (C-20), 42.1 (C-14), 42.1 (C-8), 40.4 (C-1), 39.0 (C-4), 38.0 (C-22), 37.3 (C-10), 33.2 (C-7), 29.1 (C-15), 27.8 (C-21), 27.0 (C-29), 26.8 (C-2), 26.2 (C-16), 24.7 (C-27), 24.1 (C-11), 18.8 (C-6), 17.2 (C-25), 16.7 (C-26), 16.1 (C-30), 13.2 (C-24), 62.8 (C-1'), 168.2 (C-1"). HR-ESI-MS found 561.3887. Calculated 561.3898 for $\mathrm{C}_{32} \mathrm{H}_{53} \mathrm{~N}_{2} \mathrm{O}_{6}[(\mathrm{M}+\mathrm{H})+]$.

General procedure for the preparation of benzaldehyde

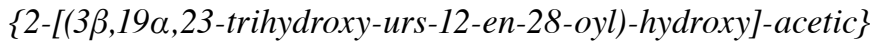
hydrazone $4 a-4 f$.

Benzaldehyde $\{2-[(3 \beta, 19 \alpha, 23$-trihydroxy-urs-12-en-28oyl)-hydroxy]-acetic\} hydrazone $\left(4 a, \mathrm{C}_{39} \mathrm{H}_{56} \mathrm{~N}_{2} \mathrm{O}_{6}, \mathrm{R}_{I}=\mathrm{C}_{6} \mathrm{H}_{5}\right.$, $\left.R_{2}=H\right)$. Compound $3(2 \mathrm{mmol})$ was dissolved in absolute EtOH $(30 \mathrm{ml})$ and benzaldehyde $(0.3 \mathrm{ml})$ was added. Glacial acetic acid $(0.2 \mathrm{ml})$ was added drop-wise and the mixture was refluxed for $8 \mathrm{~h}$. Water $(100 \mathrm{ml})$ was then added, stirred 

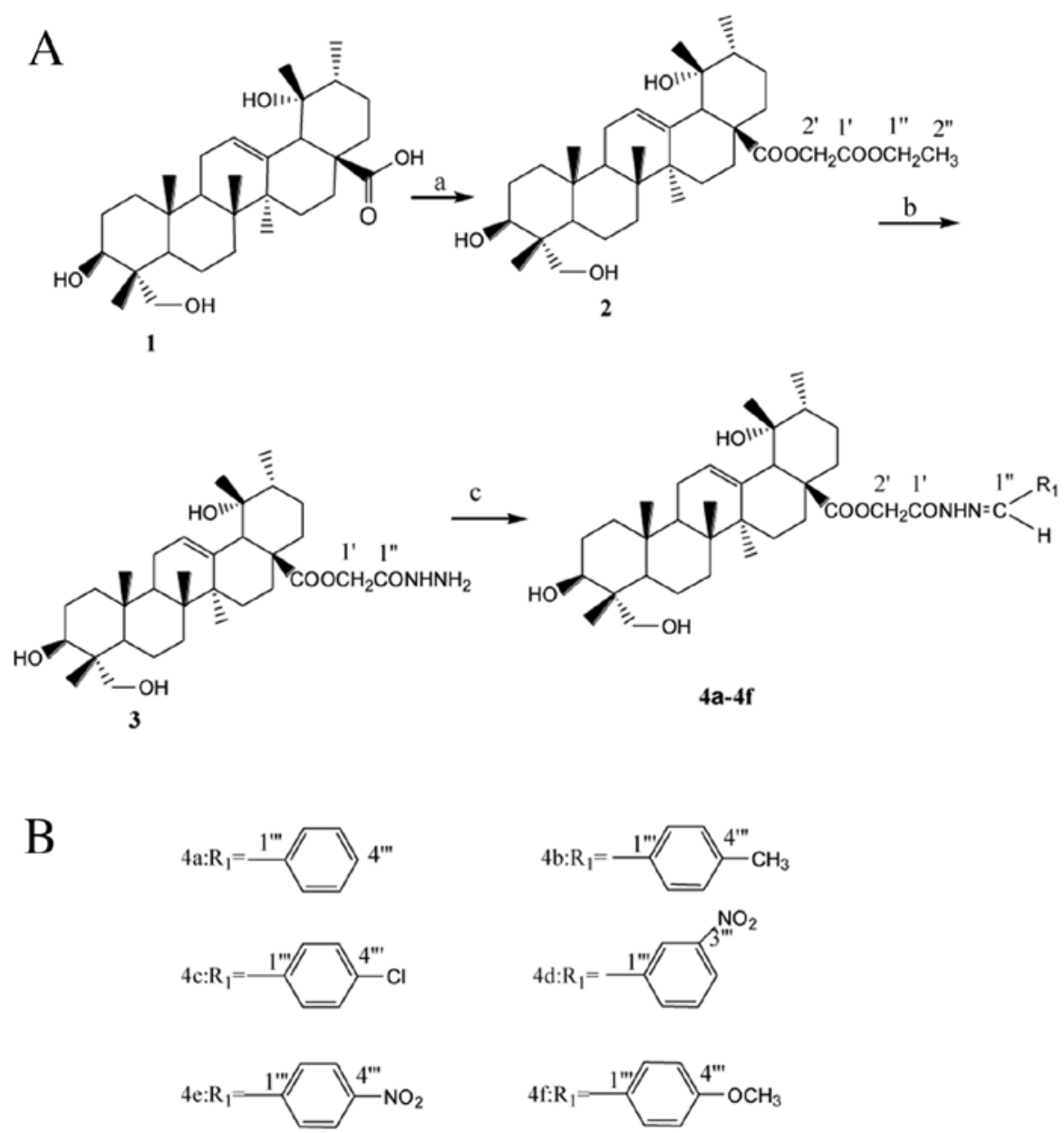

Figure 2. (A) Synthesis of RA derivatives. Reagents and conditions: (a) acetone/potassium carbonate/potassium iodide/triethylamine/ethyl chloroacetate/ refluxed/24 h; (b) hydrazine hydrate/ethanol/refluxed/11 h; (c) $\mathrm{R}_{1} \mathrm{COH} /$ glacial acetic acid/refluxed/8 h. (B) Six novel compounds $4 \mathrm{a}-4 \mathrm{f}$.

and filtered. The crude obtained was then purified on a silica gel column with petroleum ether and ethyl acetate as eluents to yield white needles. The yield was $80.3 \%$, and its melting point was between 169 and $171^{\circ} \mathrm{C} . \mathrm{IR}(\mathrm{KBr}) \mathrm{cm}^{-1}: 3619,3425$, 2929, 2876, 1693, 1449, 1388, 1204, 1140, 1047, 755 and 695; ${ }^{1} \mathrm{H}$ NMR $\left(400 \mathrm{MHz}, \mathrm{C}_{5} \mathrm{D}_{5} \mathrm{~N}\right) \delta: 12.50(1 \mathrm{H}, \mathrm{s},-\mathrm{NH}), 8.05(1 \mathrm{H}, \mathrm{s}$, H-1"), $7.64\left(2 \mathrm{H}, \mathrm{d}, J=8.0 \mathrm{~Hz}, \mathrm{H}-2 \mathrm{l}^{\prime \prime}, 6 \mathrm{6}^{\prime \prime}\right), 7.25(2 \mathrm{H}, \mathrm{t}, J=8.0 \mathrm{~Hz}$, H-3"', 5"'), 7.21 (1H, t, J=8.0 Hz, H-4"'), $5.51(2 \mathrm{H}, \mathrm{dd}, J=15.9$, $\left.26.9 \mathrm{~Hz}, \mathrm{H}-2^{\prime}\right), 5.47$ (1H, m, H-12), 4.98 (1H, m, H-3), $4.06(1 \mathrm{H}$, d, $J=10.4 \mathrm{~Hz}, \mathrm{H}-23 \mathrm{a}), 3.61(1 \mathrm{H}, \mathrm{d}, J=10.4 \mathrm{~Hz}, \mathrm{H}-23 \mathrm{~b}), 2.90(1 \mathrm{H}$, br s, $\mathrm{H}-18), 1.55$ (3H, s, $\left.\mathrm{CH}_{3}-25\right), 1.28$ (3H, s, $\left.\mathrm{CH}_{3}-27\right), 0.97$ $\left(3 \mathrm{H}, \mathrm{s}, \mathrm{CH}_{3}-29\right), 0.96\left(3 \mathrm{H}, \mathrm{d}, \mathrm{J}=6.64 \mathrm{~Hz}, \mathrm{CH}_{3}-30\right), 0.94(3 \mathrm{H}, \mathrm{s}$, $\left.\mathrm{CH}_{3}-26\right), 0.90\left(3 \mathrm{H}, \mathrm{s}, \mathrm{CH}_{3}-24\right) ;{ }^{13} \mathrm{C}$ NMR $\left(100 \mathrm{MHz}, \mathrm{C}_{5} \mathrm{D}_{5} \mathrm{~N}\right) \delta$ : 177.9 (C-28), 139.6 (C-13), 128.5 (C-12), 73.7 (C-3), 72.8 (C-19), 68.2 (C-23), 54.5 (C-18), 49.0 (C-5), 48.8 (C-9), 47.9 (C-17), 43.0 (C-20), 42.3 (C-14), 42.2 (C-8), 40.5 (C-1), 39.0 (C-4), 38.3 (C-22), 37.3 (C-10), 33.3 (C-7), 30.1 (C-15), 29.3 (C-21), 27.8 (C-29), 27.0 (C-2), 26.4 (C-16), 24.7 (C-27), 24.2 (C-11), 18.8 (C-6), 17.4 (C-25), 16.9 (C-26), 16.2 (C-30), 13.2 (C-24), 61.8 (C-2'), 169.5 (C-1'), 144.0 (C-1"), 135.0 (C-1"'), 129.2 (C-3"', 5"'), 127.6 (C-2"', 6"'), 130.3 (C-4"'). HR-ESI-MS found 649.4211. Calculated 649.4212 for $\mathrm{C}_{39} \mathrm{H}_{57} \mathrm{~N}_{2} \mathrm{O}_{6}[(\mathrm{M}+\mathrm{H})+]$.

4-methyl-benzaldehyde $\{2-[(3 \beta, 19 \alpha, 23$-trihydroxy-urs12-en-28-oyl)-hydroxy]-acetic\} hydrazone (4b, $\mathrm{C}_{40} \mathrm{H}_{58} \mathrm{~N}_{2} \mathrm{O}_{6}$,
$\left.R_{I}=\mathrm{CH}_{3} \mathrm{C}_{6} \mathrm{H}_{4}, \mathrm{R}_{2}=H\right)$. Compound 3 was reacted with p-methyl benzaldehyde using the general procedure to give compound $4 \mathrm{~b}$, which was eluted with petroleum ether/ethyl acetate $(\mathrm{V} / \mathrm{V})=1: 5$ to give white powder. The yield was $87.2 \%$ and its melting point was between 210 and $211^{\circ} \mathrm{C}$. $\mathrm{IR}(\mathrm{KBr}) \mathrm{cm}^{-1}$. $3620,3275,2928,2874,1739,1688,1468,1388,1229,1149$, 1046, 813 and 514; ${ }^{1} \mathrm{H}$ NMR $\left(400 \mathrm{MHz}, \mathrm{C}_{5} \mathrm{D}_{5} \mathrm{~N}\right) \delta: 12.43(1 \mathrm{H}$, s, -NH), $8.04\left(1 \mathrm{H}, \mathrm{s}, \mathrm{H}-1^{\prime \prime}\right), 7.57$ (2H, d, J=8.0 Hz, H-2'",6'"), $7.08\left(2 \mathrm{H}, \mathrm{d}, J=8.0 \mathrm{~Hz}, \mathrm{H}-3{ }^{\prime \prime}, 5^{\prime \prime}\right), 5.54(2 \mathrm{H}, \mathrm{dd}, J=15.8,27.0 \mathrm{~Hz}$, $\left.\mathrm{H}-2^{\prime}\right), 5.46(1 \mathrm{H}, \mathrm{m}, \mathrm{H}-12), 4.97(1 \mathrm{H}, \mathrm{m}, \mathrm{H}-3), 4.07(1 \mathrm{H}, \mathrm{d}$, $J=10.4 \mathrm{~Hz}, \mathrm{H}-23 \mathrm{a}), 3.61(1 \mathrm{H}, \mathrm{d}, J=10.4 \mathrm{~Hz}, \mathrm{H}-23 \mathrm{~b}), 2.90(1 \mathrm{H}$, br s, H-18), 2.10 (3H, s, 4"'- $\left.\mathrm{CH}_{3}\right), 1.55\left(3 \mathrm{H}, \mathrm{s}, \mathrm{CH}_{3}-25\right), 1.28$ $\left(3 \mathrm{H}, \mathrm{s}, \mathrm{CH}_{3}-27\right), 0.97\left(3 \mathrm{H}, \mathrm{s}, \mathrm{CH}_{3}-29\right), 0.96(3 \mathrm{H}, \mathrm{d}, J=6.64 \mathrm{~Hz}$, $\left.\mathrm{CH}_{3}-30\right), 0.91$ (3H, s, $\left.\mathrm{CH}_{3}-26\right), 0.89$ (3H, s, $\left.\mathrm{CH}_{3}-24\right) ;{ }^{13} \mathrm{C} \mathrm{NMR}$ $\left(100 \mathrm{MHz}, \mathrm{C}_{5} \mathrm{D}_{5} \mathrm{~N}\right)$ 8: $177.9(\mathrm{C}-28), 139.6(\mathrm{C}-13), 128.5(\mathrm{C}-12)$, 73.7 (C-3), 72.8 (C-19), 68.2 (C-23), 54.5 (C-18), 49.0 (C-5), 48.8 (C-9), 47.9 (C-17), 43.0 (C-20), 42.3 (C-14), 42.2 (C-8), 40.5 (C-1), 39.0 (C-4), 38.3 (C-22), 37.3 (C-10), 33.3 (C-7), 30.1 (C-15), 29.3 (C-21), 27.8 (C-29), 27.1 (C-2), 26.4 (C-16), 24.7 (C-27), 24.2 (C-11), 18.9 (C-6), 17.4 (C-25), 16.8 (C-26), 16.2 (C-30), 13.2 (C-24), 61.8 (C-2'), 169.4 (C-1'), 144.2 (C-1"), 132.3 (C-1"'), 129.9 (C-3'", 5"'), 127.6 (C-2"', 6"'), 140.3 (C-4'"), 21.4 $\left(4 " '-\mathrm{CH}_{3}\right)$. HR-ESI-MS found 663.4388. Calculated 663.4368 for $\mathrm{C}_{40} \mathrm{H}_{59} \mathrm{~N}_{2} \mathrm{O}_{6}[(\mathrm{M}+\mathrm{H})+]$. 
4-chloride-benzaldehyde $\{2-[(3 \beta, 19 \alpha, 23-$ trihydroxy -urs-12-en-28-oyl)-hydroxy]-acetic\} hydrazone (4c, $\mathrm{C}_{39} \mathrm{H}_{55} \mathrm{ClN}_{2} \mathrm{O}_{6}, \mathrm{R}_{1}=\mathrm{p}-\mathrm{ClC}_{6} \mathrm{H}_{4}, \mathrm{R}_{2}=\mathrm{H}$ ). Compound 3 was reacted with p-chlorine benzaldehyde using the general procedure to give compound $4 \mathrm{c}$, which was eluted with petroleum ether/ethyl acetate $(\mathrm{V} / \mathrm{V})=1: 5$ to give white needles. The yield was $91.0 \%$ and its melting point was between 205 and $207^{\circ} \mathrm{C}$. $\operatorname{IR}(\mathrm{KBr}) \mathrm{cm}^{-1}: 3620,3440,2930,2876,1699,1459,1388,1259$, 1140, 1046, 826 and 515; ${ }^{1} \mathrm{H}$ NMR (400 MHz, $\left.\mathrm{C}_{5} \mathrm{D}_{5} \mathrm{~N}\right) \mathrm{\delta}: 12.60$ $(1 \mathrm{H}, \mathrm{s},-\mathrm{NH}), 7.98(1 \mathrm{H}, \mathrm{s}, \mathrm{H}-1 "), 7.57$ (2H, d, $J=8.8 \mathrm{~Hz}, \mathrm{H}-2{ }^{\prime \prime \prime}$, 6"'), 7.31 (2H, d, $J=8.8 \mathrm{~Hz}, \mathrm{H}-3$ "', 5 "'), 5.49 (2H, dd, $J=15.8$, $\left.28.4 \mathrm{~Hz}, \mathrm{H}-2^{\prime}\right), 5.43(1 \mathrm{H}, \mathrm{m}, \mathrm{H}-12), 5.00(1 \mathrm{H}, \mathrm{m}, \mathrm{H}-3), 4.08$ $(1 \mathrm{H}, \mathrm{d}, J=10.4 \mathrm{~Hz}, \mathrm{H}-23 \mathrm{a}), 3.61(1 \mathrm{H}, \mathrm{d}, J=10.4 \mathrm{~Hz}, \mathrm{H}-23 \mathrm{~b}), 2.90$ $\left(1 \mathrm{H}\right.$, br s, H-18), $1.55\left(3 \mathrm{H}, \mathrm{s}, \mathrm{CH}_{3}-25\right), 1.28\left(3 \mathrm{H}, \mathrm{s}, \mathrm{CH}_{3}-27\right)$, $0.97\left(3 \mathrm{H}, \mathrm{s}, \mathrm{CH}_{3}-29\right), 0.96\left(3 \mathrm{H}, \mathrm{d}, J=6.64 \mathrm{~Hz}, \mathrm{CH}_{3}-30\right), 0.94$ $\left(3 \mathrm{H}, \mathrm{s}, \mathrm{CH}_{3}-26\right), 0.91\left(3 \mathrm{H}, \mathrm{s}, \mathrm{CH}_{3}-24\right) ;{ }^{13} \mathrm{C}$ NMR $(100 \mathrm{MHz}$, $\left.\mathrm{C}_{5} \mathrm{D}_{5} \mathrm{~N}\right)$ 8: $177.9(\mathrm{C}-28), 139.6(\mathrm{C}-13), 128.5(\mathrm{C}-12), 73.7$ (C-3), 72.8 (C-19), 68.2 (C-23), 54.5 (C-18), 49.0 (C-5), 48.8 (C-9), 47.9 (C-17), 43.0 (C-20), 42.3 (C-14), 42.2 (C-8), 40.5 (C-1), 39.0 (C-4), 38.3 (C-22), 37.3 (C-10), 33.3 (C-7), 30.1 (C-15), 29.3 (C-21), 27.8 (C-29), 27.1 (C-2), 26.4 (C-16), 24.7 (C-27), 24.2 (C-11), 18.9 (C-6), 17.4 (C-25), 16.8 (C-26), 16.2 (C-30), 13.2 (C-24), 61.7 (C-2'), 169.6 (C-1'), 142.6 (C-1"), 129.2 (C-1'"), 129.4 (C-3"', 5"'), 128.9 (C-2'", 6"'), 133.7 (C-4'"). HR-ESI-MS found 683.3821. Calculated 683.3821 for $\mathrm{C}_{39} \mathrm{H}_{56} \mathrm{ClN}_{2} \mathrm{O}_{6}$ $[(\mathrm{M}+\mathrm{H})+]$.

3-nitryl-benzaldehyde $\{2-[(3 \beta, 19 \alpha, 23-$ trihydroxy-urs12-en-28-oyl)-hydroxy]-acetic\} hydrazone $\left(4 d, \mathrm{C}_{39} \mathrm{H}_{55} \mathrm{~N}_{3} \mathrm{O}_{8}\right.$, $R_{1}=m-\mathrm{NO}_{2} \mathrm{C}_{6} \mathrm{H}_{4}, \mathrm{R}_{2}=\mathrm{H}$ ). Compound 3 was reacted with $\mathrm{m}$-nitryl benzaldehyde using the general procedure to give compound $4 \mathrm{~d}$, which was eluted with petroleum ether/ethyl acetate $(\mathrm{V} / \mathrm{V})=1: 5$ to give white needles. The yield was $93.0 \%$ and its melting point was between 175 and $177^{\circ} \mathrm{C}$. $\operatorname{IR}(\mathrm{KBr}) \mathrm{cm}^{-1}: 3423,2929,2876,1702,1534,1450,1388,1352$, $1228,1140,1045,736$ and $678 ;{ }^{1} \mathrm{H}$ NMR $\left(400 \mathrm{MHz}, \mathrm{C}_{5} \mathrm{D}_{5} \mathrm{~N}\right)$

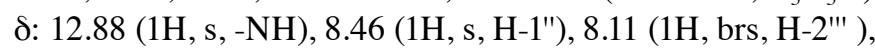
$8.06\left(1 \mathrm{H}, \mathrm{d}, J=8.0 \mathrm{~Hz}, \mathrm{H}-4^{\prime \prime \prime}\right), 8.06\left(1 \mathrm{H}, \mathrm{d}, J=8.0 \mathrm{~Hz}, \mathrm{H}-5^{\prime \prime \prime}\right), 7.37$ $\left(1 \mathrm{H}, \mathrm{t}, J=8.0 \mathrm{~Hz}, \mathrm{H}-6^{\prime \prime}\right), 5.52\left(2 \mathrm{H}, \mathrm{dd}, J=15.8,24.3 \mathrm{~Hz}, \mathrm{H}-2^{\prime}\right)$, $5.46(1 \mathrm{H}, \mathrm{m}, \mathrm{H}-12), 4.97(1 \mathrm{H}, \mathrm{m}, \mathrm{H}-3), 4.08(1 \mathrm{H}, \mathrm{d}, J=10.4 \mathrm{~Hz}$, H-23a), 3.61 (1H, d, J=10.4 Hz, H-23b), 2.90 (1H, br s, H-18), $1.55\left(3 \mathrm{H}, \mathrm{s}, \mathrm{CH}_{3}-25\right), 1.29\left(3 \mathrm{H}, \mathrm{s}, \mathrm{CH}_{3}-27\right), 0.97\left(3 \mathrm{H}, \mathrm{s}, \mathrm{CH}_{3}-29\right)$, $0.96\left(3 \mathrm{H}, \mathrm{d}, J=6.64 \mathrm{~Hz}, \mathrm{CH}_{3}-30\right), 0.96\left(3 \mathrm{H}, \mathrm{s}, \mathrm{CH}_{3}-26\right), 0.92$ (3H, s, $\left.\mathrm{CH}_{3}-24\right) ;{ }^{13} \mathrm{C}$ NMR (100 MHz, $\left.\mathrm{C}_{5} \mathrm{D}_{5} \mathrm{~N}\right) \delta: 180.0(\mathrm{C}-28)$, 139.6 (C-13), 128.5 (C-12), 73.7 (C-3), 72.8 (C-19), 68.2 (C-23), 54.6 (C-18), 49.0 (C-5), 48.8 (C-9), 47.9 (C-17), 43.0 (C-20), 42.3 (C-14), 42.2 (C-8), 40.5 (C-1), 39.0 (C-4), 38.3 (C-22), 37.3 (C-10), 33.3 (C-7), 30.1 (C-15), 29.3 (C-21), 27.8 (C-29), 27.1 (C-2), 26.5 (C-16), 24.7 (C-27), 24.2 (C-11), 18.9 (C-6), 17.4 (C-25), 16.8 (C-26), 16.2 (C-30), 13.2 (C-24), 61.7 (C-2'), 169.8 (C-1'), 141.3 (C-1"), 149.1 (C-3'"), 136.9 (C-1"'), 132.6 (C-6"'), 130.2 (C-5"'), 124.5 (C-4"'), 122.3 (C-2"'). HR-ESI-MS found 694.4062. Calculated 694.4062 for $\mathrm{C}_{39} \mathrm{H}_{56} \mathrm{~N}_{3} \mathrm{O}_{8}[(\mathrm{M}+\mathrm{H})+]$.

4-nitryl-benzaldehyde $\{2-[(3 \beta, 19 \alpha, 23$-trihydroxy-urs12-en-28-oyl)-hydroxy]-acetic\} hydrazone $\left(4 e, \mathrm{C}_{39} \mathrm{H}_{55} \mathrm{~N}_{3} \mathrm{O}_{8}\right.$, $R_{1}=p-\mathrm{NO}_{2} \mathrm{C}_{6} \mathrm{H}_{4}, \mathrm{R}_{2}=\mathrm{H}$ ). Compound 3 was reacted with p-nitryl benzaldehyde using the general procedure to give compound $4 \mathrm{e}$, which was eluted with petroleum ether/ethyl acetate $(\mathrm{V} / \mathrm{V})=1: 5$ to give pale yellow needles. The yield was $85.1 \%$ and its melting point was between 230 and $231^{\circ} \mathrm{C}$. $\operatorname{IR}(\mathrm{KBr}) \mathrm{cm}^{-1}: 3606,3499,2925,2879,1713,1699,1517,1407$,
1341, 1234, 1151, 1043, 835 and 752; ${ }^{1} \mathrm{H}$ NMR $(400 \mathrm{MHz}$, $\left.\mathrm{C}_{5} \mathrm{D}_{5} \mathrm{~N}\right) \delta: 12.96(1 \mathrm{H}, \mathrm{s},-\mathrm{NH}), 8.13(2 \mathrm{H}, \mathrm{d}, J=8.8 \mathrm{~Hz}, \mathrm{H}-2$ "',6"'), $8.06\left(1 \mathrm{H}, \mathrm{s}, \mathrm{H}-1^{\prime \prime}\right), 7.75\left(2 \mathrm{H}, \mathrm{d}, \mathrm{J}=8.8 \mathrm{~Hz}, \mathrm{H}-3^{\prime \prime \prime}, 5^{\prime \prime \prime}\right), 5.52(2 \mathrm{H}$, dd, $\left.J=15.9,26.8 \mathrm{~Hz}, \mathrm{H}-2^{\prime}\right), 5.47(1 \mathrm{H}, \mathrm{m}, \mathrm{H}-12), 5.10(1 \mathrm{H}, \mathrm{m}$, H-3), $4.08(1 \mathrm{H}, \mathrm{d}, J=10.4 \mathrm{~Hz}, \mathrm{H}-23 \mathrm{a}), 3.61(1 \mathrm{H}, \mathrm{d}, J=10.4 \mathrm{~Hz}$, $\mathrm{H}-23 \mathrm{~b}), 2.90\left(1 \mathrm{H}\right.$, br s, H-18), $1.55\left(3 \mathrm{H}, \mathrm{s}, \mathrm{CH}_{3}-25\right), 1.29(3 \mathrm{H}$, $\left.\mathrm{s}, \mathrm{CH}_{3}-27\right), 0.97\left(3 \mathrm{H}, \mathrm{s}, \mathrm{CH}_{3}-29\right), 0.96(3 \mathrm{H}, \mathrm{d}, J=6.64 \mathrm{~Hz}$, $\left.\mathrm{CH}_{3}-30\right), 0.96\left(3 \mathrm{H}, \mathrm{s}, \mathrm{CH}_{3}-26\right), 0.91\left(3 \mathrm{H}, \mathrm{s}, \mathrm{CH}_{3}-24\right) ;{ }^{13} \mathrm{C}$ NMR $\left(100 \mathrm{MHz}, \mathrm{C}_{5} \mathrm{D}_{5} \mathrm{~N}\right)$ 8: $180.0(\mathrm{C}-28), 139.6(\mathrm{C}-13), 128.5$ (C-12), 73.7 (C-3), 72.8 (C-19), 68.1 (C-23), 54.5 (C-18), 49.0 (C-5), 48.8 (C-9), 47.9 (C-17), 43.0 (C-20), 42.3 (C-14), 42.2 (C-8), 40.5 (C-1), 39.0 (C-4), 38.3 (C-22), 37.3 (C-10), 33.3 (C-7), 30.1 (C-15), 29.3 (C-21), 27.8 (C-29), 27.1 (C-2), 26.4 (C-16), 24.7 (C-27), 24.2 (C-11), 18.9 (C-6), 17.4 (C-25), 16.8 (C-26), 16.2 (C-30), 13.2 (C-24), 61.7 (C-2'), 169.6 (C-1'), 141.3 (C-1"), 140.9 (C-1'"'), 128.0 (C-3"', 5"'), 124.4 (C-2'", 6"'), 148.6 (C-4"'). HR-ESI-MS found 694.4061. Calculated 694.4062 for $\mathrm{C}_{39} \mathrm{H}_{56} \mathrm{~N}_{3} \mathrm{O}_{8}[(\mathrm{M}+\mathrm{H})+]$.

4-methoxy-benzaldehyde $\{2-[(3 \beta, 19 \alpha, 23$-trihydroxy-urs12-en-28-oyl)-hydroxy]-acetic\} hydrazone ( $4 f, \mathrm{C}_{40} \mathrm{H}_{58} \mathrm{~N}_{2} \mathrm{O}_{7}$, $\left.R_{1}=p-\mathrm{CH}_{3} \mathrm{OC}_{6} \mathrm{H}_{4}, \mathrm{R}_{2}=\mathrm{H}\right)$. Compound 3 was reacted with p-methoxy benzaldehyde using the general procedure to give compound 4f, which was eluted with petroleum ether/ethyl acetate $(\mathrm{V} / \mathrm{V})=1: 3$ to give white needles. The yield was $89.2 \%$ and its melting point was between 173 and $175^{\circ} \mathrm{C}$. IR $(\mathrm{KBr}) \mathrm{cm}^{-1}$ : 3622, 3483, 2932, 2875, 1688, 1607, 1516, 1462, 1377, 1252, $1170,1034,831$ and $530 ;{ }^{1} \mathrm{H}$ NMR $\left(400 \mathrm{MHz}, \mathrm{C}_{5} \mathrm{D}_{5} \mathrm{~N}\right) \delta: 12.40$ $(1 \mathrm{H}, \mathrm{s},-\mathrm{NH}), 8.04(1 \mathrm{H}, \mathrm{s}, \mathrm{H}-1 "), 7.63\left(2 \mathrm{H}, \mathrm{d}, J=8.8 \mathrm{~Hz}, \mathrm{H}-2^{\prime \prime \prime}\right.$, $\left.6^{\prime \prime \prime}\right), 6.87\left(2 \mathrm{H}, \mathrm{d}, J=8.8 \mathrm{~Hz}, \mathrm{H}-3^{\prime \prime \prime}, 5^{\prime \prime \prime}\right), 5.49$ (2H, dd, $J=15.9$, $\left.28.2 \mathrm{~Hz}, \mathrm{H}-2^{\prime}\right), 5.43(1 \mathrm{H}, \mathrm{m}, \mathrm{H}-12), 4.99(1 \mathrm{H}, \mathrm{m}, \mathrm{H}-3), 4.08(1 \mathrm{H}$, d, $J=10.4 \mathrm{~Hz}, \mathrm{H}-23 \mathrm{a}), 3.61(1 \mathrm{H}, \mathrm{d}, J=10.4 \mathrm{~Hz}, \mathrm{H}-23 \mathrm{~b}), 3.56(3 \mathrm{H}$, $\left.\mathrm{s},-\mathrm{OCH}_{3}\right), 2.90(1 \mathrm{H}$, br s, $\mathrm{H}-18), 1.55\left(3 \mathrm{H}, \mathrm{s}, \mathrm{CH}_{3}-25\right), 1.28$ $\left(3 \mathrm{H}, \mathrm{s}, \mathrm{CH}_{3}-27\right), 0.97\left(3 \mathrm{H}, \mathrm{s}, \mathrm{CH}_{3}-29\right), 0.96(3 \mathrm{H}, \mathrm{d}, J=6.64 \mathrm{~Hz}$, $\left.\mathrm{CH}_{3}-30\right), 0.94\left(3 \mathrm{H}, \mathrm{s}, \mathrm{CH}_{3}-26\right), 0.90\left(3 \mathrm{H}, \mathrm{s}, \mathrm{CH}_{3}-24\right) ;{ }^{13} \mathrm{C} \mathrm{NMR}$ $\left(100 \mathrm{MHz}, \mathrm{C}_{5} \mathrm{D}_{5} \mathrm{~N}\right)$ ठ: $177.9(\mathrm{C}-28), 139.6(\mathrm{C}-13), 128.5(\mathrm{C}-12)$, 73.7 (C-3), 72.8 (C-19), 68.2 (C-23), 54.5 (C-18), 49.0 (C-5), 48.8 (C-9), 47.9 (C-17), 43.0 (C-20), 42.3 (C-14), 42.2 (C-8), 40.5 (C-1), 39.0 (C-4), 38.3 (C-22), 37.3 (C-10), 33.3 (C-7), 30.1 (C-15), 29.3 (C-21), 27.8 (C-29), 27.1 (C-2), 26.4 (C-16), 24.7 (C-27), 24.2 (C-11), 18.9 (C-6), 17.4 (C-25), 16.8 (C-26), 16.2 (C-30), 13.2 (C-24), 62.8 (C-2'), 169.3 (C-1'), 143.9 (C-1"), 127.7 (C-1"'), 129.2 (C-3"', 5"'), 114.8 (C-2"', 6"'), 161.7 (C-4"'), 55.4 $\left(\mathrm{C}-\mathrm{OCH}_{3}\right)$. HR-ESI-MS found 679.4332. Calculated 679.4317 for $\mathrm{C}_{40} \mathrm{H}_{59} \mathrm{~N}_{2} \mathrm{O}_{7}[(\mathrm{M}+\mathrm{H})+]$.

Cell cytotoxicity assays. The cell survival rate was measured by an MTT assay (29). Aliquots ( $200 \mu \mathrm{l})$ of $5 \times 10^{3}$ cells $/ \mathrm{ml}$ of HeLa, HepG2 and SGC-7901 cells were seeded in 96-well plates in DMEM medium containing $10 \% \mathrm{FBS}$ at $37^{\circ} \mathrm{C}$ in a humidified atmosphere of $5 \% \mathrm{CO}_{2}$. The test drugs were dissolved in dimethyl sulfoxide (DMSO). The incubation medium was replaced with each test medium, making a final concentration of 1.25 to $100 \mu \mathrm{M}$ of RA and compounds $4 \mathrm{a}-4 \mathrm{f}$ for $48 \mathrm{~h}$ and DMSO at $0.1 \%$ in media as a vehicle control. After adding $5 \mu \mathrm{l}$ of MTT labeling reagent (MTT Cell Proliferation Assay kit; Trevigen, USA) to each well, the plates were incubated for $4 \mathrm{~h}$, before each well was supplemented with $100 \mu \mathrm{l}$ solubilization solution. The absorbance at $570 \mathrm{~nm}$ was then measured in a microtiter plate reader (Bio-Tek, Winooski, VT, USA). The drug treatments were performed separately three times. 
Table I. The $\mathrm{IC}_{50}$ values of $\mathrm{RA}$ and its derivatives $4 \mathrm{a}-4 \mathrm{f}$ on human cancer cell lines.

\begin{tabular}{lccr}
\hline & & $\mathrm{IC}_{50}(\mu \mathrm{M})$ & SGC-7901 \\
\cline { 2 - 4 } Compound & HeLa & HepG2 & $16.48 \pm 2.32$ \\
\hline RA & $18.70 \pm 1.61$ & $8.26 \pm 1.24$ & $44.29 \pm 4.27$ \\
$4 \mathrm{a}$ & $86.67 \pm 3.86$ & $>100^{\mathrm{a}}$ & $41.22 \pm 2.98$ \\
$4 \mathrm{~b}$ & $20.58 \pm 0.79$ & $34.60 \pm 3.55$ & $>100^{\mathrm{a}}$ \\
$4 \mathrm{c}$ & $>100^{\mathrm{a}}$ & $>100^{\mathrm{a}}$ & $>100^{\mathrm{a}}$ \\
$4 \mathrm{~d}$ & $9.58 \pm 1.14^{\mathrm{b}}$ & $45.36 \pm 3.36$ & $15.38 \pm 1.58$ \\
$4 \mathrm{e}$ & $18.83 \pm 2.26$ & $8.74 \pm 1.28$ & $11.32 \pm 1.20^{\mathrm{b}}$ \\
$4 \mathrm{f}$ & $8.54 \pm 0.97^{\mathrm{b}}$ & $4.16 \pm 0.73^{\mathrm{b}}$ & \\
\hline
\end{tabular}

Data are represented as means $\pm \mathrm{SD} ; \mathrm{n}=3$. ${ }^{\mathrm{a}} \mathrm{IC}_{50}$ values $>100 \mu \mathrm{M}$ are indicated as $>100$; ${ }^{\mathrm{b}} \mathrm{P}<0.05$ vs. RA.

Apoptosis determination by DAPI staining. Apoptotic morphological changes were determined by DAPI staining. The harvested cells were exposed with compound $4 \mathrm{f}$ for $48 \mathrm{~h}$. The medium was then removed from the culture, and the cells were washed twice with cold PBS and fixed with $100 \% \mathrm{EtOH}$ for $20 \mathrm{~min}$ at room temperature. It was washed twice again with PBS and incubated with DAPI solution $(0.5 \mu \mathrm{g} / \mathrm{ml})$ for $30 \mathrm{~min}$. Then, cell morphology was evaluated by fluorescence microscopy (IX70-SIF2; Olympus).

Annexin V-FITC and PI double staining analysis by flow cytometry. The cells were plated at appropriate densities $\left(\sim 2.5 \times 10^{4}\right.$ cells/well $)$ in $3 \mathrm{ml}$ of medium in 6 -well plates. The induction of apoptosis was evaluated in HepG2 cells plated on 6-well plates overnight with compound $4 \mathrm{f}$ dissolved in DMEM medium. After $48 \mathrm{~h}$ in culture, the adherent cells were harvested, washed twice with cold PBS, and then assayed for apoptosis by double staining with Annexin V-FITC and PI (Annexin V-FITC Apoptosis Detection kit; KeyGEN Biotech, Nanking, China). Briefly, $5 \times 10^{5}$ cells were re-suspended in $1 \mathrm{x}$ binding buffer and stained with $5 \mu \mathrm{l}$ of FITC-Annexin V. After 10 min of incubation, $5 \mu \mathrm{l}$ of PI was added, and the samples were incubated again for $15 \mathrm{~min}$. The samples were then immediately analyzed using a flow cytometer (FACScan; BD Biosciences, Milan, Italy) with a dedicated software. The cells in the upper right portion were late-apoptotic cells. The cells in the lower left portion were viable cells. The cells in the lower right portion were early apoptotic cells.

Cell cycle analysis. HepG 2 cells $\left(2 \times 10^{5} /\right.$ well) were placed in 6 -well plates, and then compound $4 \mathrm{f}$ was added to the wells and cultured for $48 \mathrm{~h}$. The vehicle control in the media was DMSO at $0.1 \%$. The cells from each well were harvested individually by centrifugation. The isolated cells were fixed by $70 \% \mathrm{EtOH}$ at $4{ }^{\circ} \mathrm{C}$ overnight, and then re-suspended in PBS containing $40 \mu \mathrm{g} / \mathrm{ml} \mathrm{PI}, 0.1 \mathrm{mg} / \mathrm{ml}$ RNase A, and $0.1 \%$ Triton $\mathrm{X}-100$ in a dark room for $30 \mathrm{~min}$ at $37^{\circ} \mathrm{C}$. The DNA content of 20,000 cells for each determination was measured using EPICS XL-MCL flow cytometer (Beckman Coulter, Inc., Fullerton, CA, USA), in which an argon laser $(488 \mathrm{~nm})$ was used to excite PI. An emission above $550 \mathrm{~nm}$ was collected.
Statistical analysis. The data were analyzed for mean values and standard deviation for all the experiments. Statistically significant differences were determined between the control and treated groups using Student's t-test. $\mathrm{P}<0.05$ was considered to indicate a statistically significant difference. Statistical package for the Social Sciences (SPSS, version 13.0) was used for statistical analyses.

\section{Results}

Preparation of $R A$. The barks of I. rotunda were shade-dried, grounded, and extracted with refluxing $80 \% \mathrm{EtOH}$. The airdried and powdered total saponins from EtOH extract were hydrolyzed by $4 \%$ sodium hydroxide in $30 \% \mathrm{EtOH}$ and purified by recrystallization to prepare RA. The purity of RA used was found to be $\geq 98 \%$ in a high performance liquid chromatography assay.

Structure modification of RA. The 28-COOH position of RA was modified to obtain six new compounds (Fig. 2). The cytotoxic activity of RA and its six derivatives were studied.

Cell cytotoxicity assays. The effects of RA and its derivatives $4 \mathrm{a}-4 \mathrm{f}$ on cell cytotoxicity were measured by MTT assay. The three types of human cancer cell lines were exposed to 1.25 to $100 \mu \mathrm{M}$ of RA derivatives for $48 \mathrm{~h}$. RA showed significant $\mathrm{IC}_{50}$ values of $18.70,8.26$ and $16.48 \mu \mathrm{M}$ on the HeLa, HepG2 and SGC-7901 cell lines, respectively (Table I).

Apoptosis determination by 4'-6-diamidino-2-phenylindole (DAPI) staining. HepG2 cells were exposed to compound $4 \mathrm{f}$ in its $\mathrm{IC}_{50}(4.16 \mu \mathrm{M})$ for $48 \mathrm{~h}$. The occurrence of nuclear condensation upon treatment with compound $4 \mathrm{f}$ could be revealed by DAPI staining. Apoptotic bodies, one of the stringent morphological criteria of apoptosis, were present in the compound 4f-treated HepG2 cells stained with DAPI (Fig. 3).

Annexin V-FITC assay. HepG2 cells were treated with compound $4 \mathrm{f}$ at a concentration $4.16 \mu \mathrm{M}$ for $48 \mathrm{~h}$ and were analyzed by flow cytometry. As shown in Fig. 4A, the numbers of early and late apoptotic cells were significantly increased compared to the control group. The proportion of early and late 


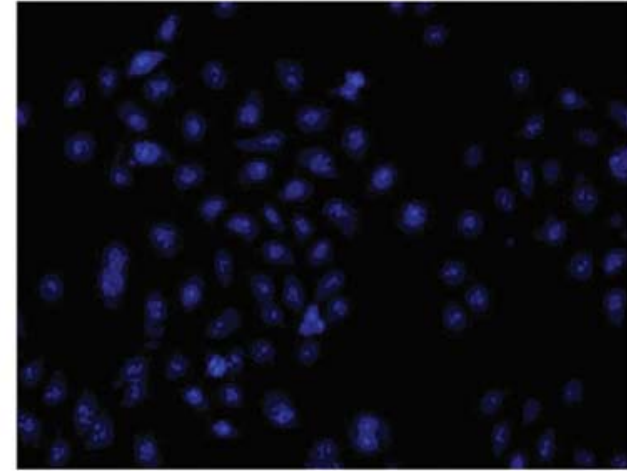

Control

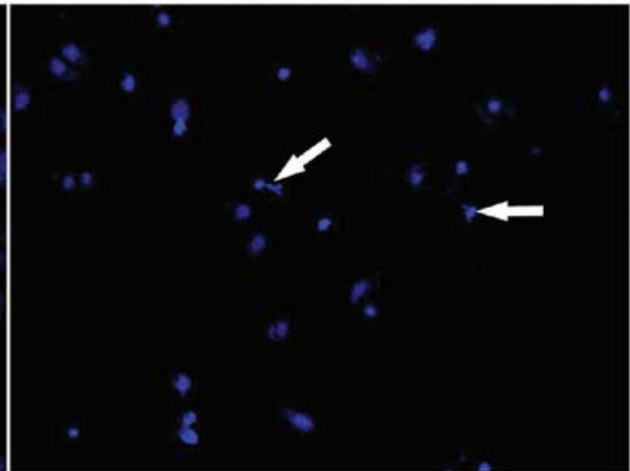

Compound $4 f$

Figure 3. DAPI staining of compound 4f-treated HepG2 cells. Cells were exposed to either control ( $0.1 \%$ DMSO in medium) or compound $4 \mathrm{f}$ at $4.16 \mu \mathrm{M}$ and incubated for $48 \mathrm{~h}$. (Magnification, x200).
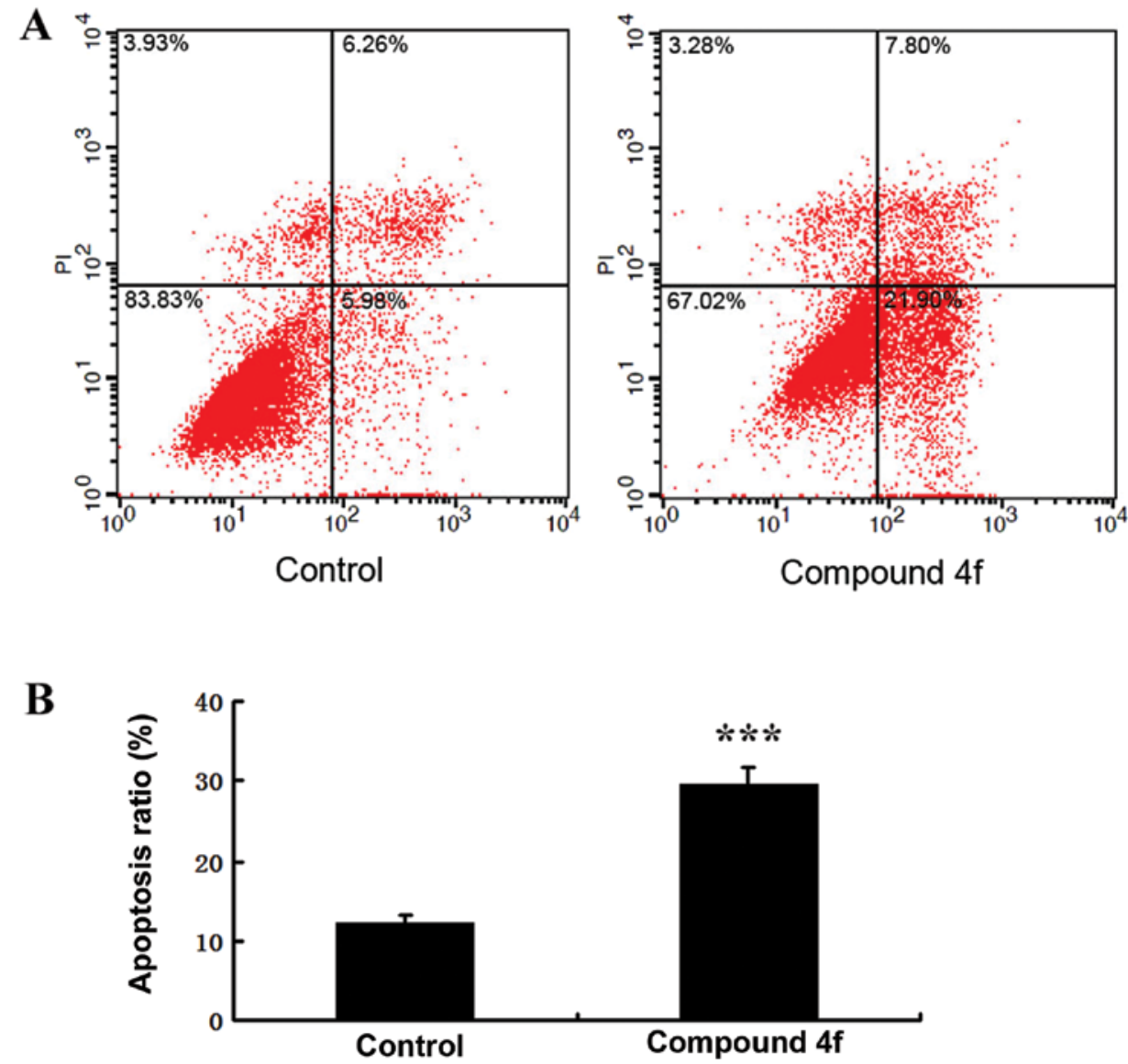

Figure 4. Apoptosis detection, by flow cytometry, of HepG2 cells treated with compound 4f. (A) Flow cytometric analysis of apoptosis in HepG2 cells treated with compound $4 \mathrm{f}$. Cells were exposed to either control (0.1\% DMSO in medium) or compound $4 \mathrm{f}$ at $4.16 \mu \mathrm{M}$ and incubated for $48 \mathrm{~h}$. The experiments were repeated at least three times. (B) Percentage of apoptotic cells. The apoptotic cells were calculated as the percentage of apoptotic cells in the upper right portion and lower right portion to the total number of the cells. Data are expressed as the means \pm SD of three experiments $\left({ }^{* * * *} \mathrm{P}<0.001 \mathrm{vs}\right.$. control).

apoptotic cells in the compound $4 \mathrm{f}$ treatment group reached $30.38 \%$, which was higher than the control group (12.5\%) $(\mathrm{P}<0.001)$ (Fig. 4B). This finding suggested that compound $4 \mathrm{f}$ suppressed cell proliferation possibly by inducing apoptosis.

Compound $4 f$ causes G0/G1 cell cycle arrest in HepG2 cells. As shown in Fig. 5A, compared with the control group, compound $4 \mathrm{f}$ resulted in a significant accumulation of $\mathrm{HepG} 2$ cells in the G0/G1 phase and a decrease in the number of cells in the G2/M phase. Markedly, more HepG2 cells treated by compound $4 \mathrm{f}$ were in the G1 phase compared to the control (78.97 vs. $65.49 \% ; \mathrm{P}<0.05$ ). These results indicated that compound $4 \mathrm{f}$ caused cell cycle arrest in the $\mathrm{G} 0 / \mathrm{G} 1$ phase (Fig. 5B). 
A

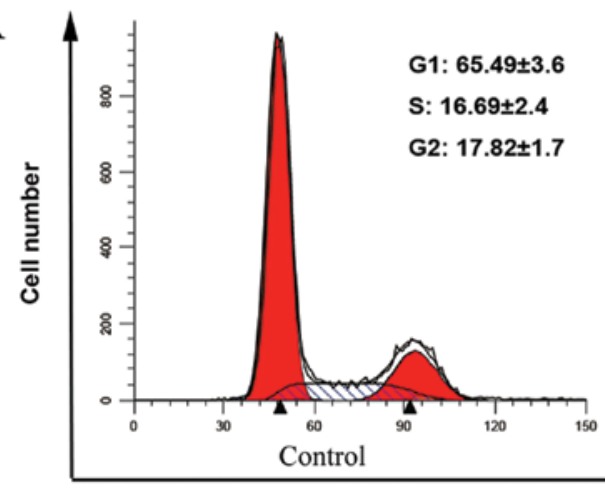

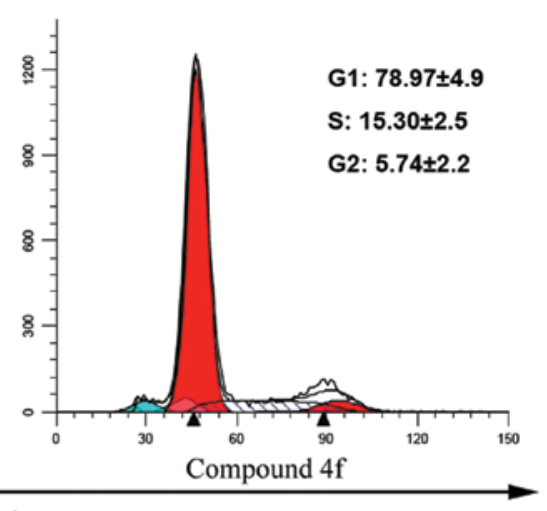

DA content

B

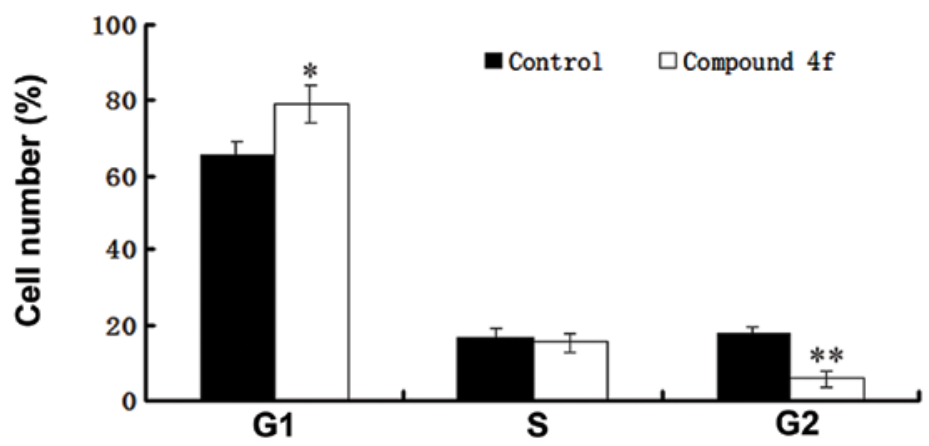

Figure 5. Compound 4f induces cell cycle arrest of HepG2 cells in the G0/G1 phase. (A) Effects of compound 4f on cell cycle distribution of HepG2 cells after staining with PI. Cells were exposed to either control (0.1\% DMSO in medium) or compound $4 \mathrm{f}$ at $4.16 \mu \mathrm{M}$ and incubated for $48 \mathrm{~h}$. (B) The percentage of cells in each phase of the cell cycle. The columns or points represent the means \pm SD of three independent experiments. ${ }^{*} \mathrm{P}<0.05,{ }^{* *} \mathrm{P}<0.01 \mathrm{vs}$. control.

\section{Discussion}

It has been reported that structural modifications could enhance the anticancer activities of parent compounds $(19,20)$. In the present study, the $28-\mathrm{COOH}$ position of RA was modified, and six new compounds were obtained. The synthesized derivatives were tested for antitumor activities in order to test previous evidence that the amino acid modification may enhance the antitumor activities of the parent (21).

The cytotoxicity results of the RA derivatives, compounds 4a-4f, demonstrated that the $\mathrm{IC}_{50}$ of compound $4 \mathrm{f}$ was significantly less than the groups treated with compounds $4 \mathrm{c}$ and $4 \mathrm{e}$. The results may be explained by the difference of substituent group in para-position of benzene of $\mathrm{R}_{1}$ group. Antitumor activities of these compounds were enhanced when they were substituted with electron-donating groups on para-position of benzene. In addition, the antitumor activity of compound $4 \mathrm{f}$ was found to be better than $4 \mathrm{~b}$. Hence, compound $4 \mathrm{f}$ was substituted with a methoxy group (a stronger electron-donation group) on para-position of benzene of $R_{1}$ group. On the other hand, since the compounds $4 \mathrm{c}, 4 \mathrm{~d}$ and $4 \mathrm{e}$ were substituted with electronattracting groups, their antitumor activities were weaker than RA. Based on the results, the $\mathrm{IC}_{50}$ of compound $4 \mathrm{f}$ was 8.54, 4.16 and $11.32 \mu \mathrm{M}$ on the HeLa, HepG2 and SGC-7901 cell lines, respectively. This indicated that compound $4 \mathrm{f}$ could be further studied as a novel antitumor agent.

Evasion of apoptosis is one of the major hallmarks of cancer and a target for cancer treatment (22). Previous data suggested apoptosis as an underlying mechanism, by which various anticancer and chemopreventive agents exert their antitumor effects $(23,24)$. The current study included the preliminary research on apoptosis and cell cycle. In the early stages of apoptosis, phosphatidylserine in the cell membrane was translocated outside. Compound $4 \mathrm{f}$ was able to induce apoptosis of HepG2 cells, and the apoptosis ratio in the early stage was about three times higher than in the control group.

The results of fluorescence-activated cell sorting of HepG2 cells treated with compound $4 \mathrm{f}$ at its $\mathrm{IC}_{50}$ showed significant cell cycle arrest at the G0/G1 interface, suggesting this as a mechanism for the antiproliferative effect of compound $4 \mathrm{f}$. However, further mechanistic investigation of compound $4 \mathrm{f}$ remains to be conducted. These findings will provide new insight into the cancer chemotherapeutic properties of RA and its derivatives.

In conclusion, six novel RA derivatives were synthesized and evaluated for their cytotoxic properties on three tumor cell lines. Compound $4 \mathrm{f}$ had a substantially better antitumor effect than RA in vitro on HepG2 cells. Compound $4 \mathrm{f}$ induced apoptosis in HepG2 cells and G0/G1 cell cycle arrest. These results provide further insight into compound $4 \mathrm{f}$-induced apoptosis and deepen our previous knowledge of the cytotoxicity and anticancer ability of RA derivatives.

\section{Acknowledgements}

The authors thank Professor Richard Nicholson at the University of Newcastle, Australia, for editing this manuscript and providing valuable suggestions. 


\section{References}

1. Parkin DM, Bray F, Ferlay J and Pisani P: Estimating the world cancer burden: Globocan 2000. Int J Cancer 94: 153-156, 2001.

2. El-Serag HB and Mason AC: Rising incidence of hepatocellular carcinoma in the United States. N Engl J Med 340: 745-750, 1999.

3. Parkin DM, Bray F, Ferlay $\mathbf{J}$ and Pisani P: Global cancer statistics, 2002. CA Cancer J Clin 55: 74-108, 2005.

4. Hwang JP and Hassan MM: Survival and hepatitis status among Asian Americans with hepatocellular carcinoma treated without liver transplantation. BMC Cancer 9: 46, 2009.

5. Charette N, De Saeger C, Lannoy V, Horsmans Y, Leclercq I and Starkel P: Salirasib inhibits the growth of hepatocarcinoma cell lines in vitro and tumor growth in vivo through ras and mTOR inhibition. Mol Cancer 9: 256, 2010.

6. Bruix J, Hessheimer AJ, Forner A, Boix L, Vilana R and Llovet JM: New aspects of diagnosis and therapy of hepatocellular carcinoma. Oncogene 25: 3848-3856, 2006.

7. Thang TD, Kuo PC, Yu CS, et al: Chemical constituents of the leaves of Glochidion obliquum and their bioactivity. Arch Pharm Res 34: 383-389, 2011.

8. Haraguchi H, Kataoka S, Okamoto S, Hanafi M and Shibata K: Antimicrobial triterpenes from Ilex integra and the mechanism of antifungal action. Phytother Res 13: 151-156, 1999.

9. Zhao HR, Wang MS and Zhou GP: Studies on constituents of Ilex chinensis Sims. Zhongguo Zhong Yao Za Zhi 18: 226-228, 255, 1993 (In Chinese).

10. Sun H, Zhang XQ, Cai Y, Han WL, Wang Y and Ye WC: Study on chemical constituents of Ilex rotunda Thunb. Chem Indus Forest Prod 295: 111-114, 2009 (In Chinese).

11. Zhao WM, Wolfender JL, Hostettmann K, Cheng KF, Xu RS and Qin GW: Triterpenes and triterpenoid saponins from Mussaenda pubescens. Phytochemistry 45: 1073-1078, 1997.

12. Saimaru H, Orihara Y, Tansakul P, Kang YH, Shibuya M and Ebizuka Y: Production of triterpene acids by cell suspension cultures of Olea europaea. Chem Pharm Bull (Tokyo) 55: 784-788, 2007

13. Lee TH, Juang SH, Hsu FL and Wu CY: Triterpene acids from the leaves of Planchonella duclitan (Blanco) Bakhuizan. J Chin Chem Sci 52: 1275-1280, 2005.

14. Zhao QC, Nan ML, He YF and Chen SW: Application of rotundic acid in the preparation of lipid-lowering drugs. CHN 201010204607.3, 2010 (In Chinese).

15. He YF, Zhao QC, Nan ML, et al: Application of rotundic acid and its derivatives in the preparation of anticancer drugs. CHN 201010607515.x, 2010 (In Chinese).
16. Zhao QC, He YF, Nan ML, Chen SW, Zhao YW and Wang LP: Synthesis method of rotundic acid derivatives and their application in the preparation of cardiovascular disease prevention Drugs. CHN 20110030007.4, 2011 (In Chinese).

17. He YF, Nan ML, Zhao QC, Zhao YW and Yue FG: Application of amino acid modified rotundic acid derivatives in the preparation of anticancer drugs. CHN 201110351365.5, 2011 (In Chinese).

18. He YF, Nan ML, Sun JM, et al: Synthesis, characterization and cytotoxicity of new rotundic acid derivatives. Molecules 17: 1278-1291, 2012.

19. Scrimin F, Becagli L and Ambrosini A: Malignant melanoma of the vulva. Considerations on 4 cases. Minerva Ginecol 38: 43-48, 1986 (In Italian).

20. Wang X, Lu N, Yang Q, et al: Studies on chemical modification and biology of a natural product, gambogic acid (III): determination of the essential pharmacophore for biological activity. Eur J Med Chem 46: 1280-1290, 2011.

21. Xu R: Guangzhou University of Chinese Medicine, 2009.

22. Abbott RG, Forrest S and Pienta KJ: Simulating the hallmarks of cancer. Artif Life 12: 617-634, 2006.

23. Sandur SK, Ichikawa H, Sethi G, Ahn KS and Aggarwal BB: Plumbagin (5-hydroxy-2-methyl-1,4-naphthoquinone) suppresses NF-kappaB activation and NF-kappaB-regulated gene products through modulation of p65 and IkappaBalpha kinase activation, leading to potentiation of apoptosis induced by cytokine and chemotherapeutic agents. J Biol Chem 281: 17023-17033, 2006.

24. Park C, Jin CY, Kwon HJ, et al: Induction of apoptosis by esculetin in human leukemia U937 cells: roles of Bcl-2 and extracellular-regulated kinase signaling. Toxicol In Vitro 24: 486-494, 2010.

25. Kim NC, Desjardins AE, Wu CD and Kinghorn AD: Activity of triterpenoid glycosides from the root bark of Mussaenda macrophylla against two oral pathogens. J Nat Prod 62: 1379-1384, 1999.

26. Zhang AL, Ye Q, Li BG, Qi HY and Zhang GL: Phenolic and triterpene glycosides from the stems of Ilex litseaefolia. J Nat Prod 68: 1531-1535, 2005.

27. Xie GB, Zhou SX, Lu YN, Lei LD and Tu PF: Triterpenoid glycosides from the leaves of Ilex pernyi. Chem Pharm Bull (Tokyo) 57: 520-524, 2009

28. Nakatani M, Hatanaka S, Komura H, Kubota T and Hase T: The structure of rotungenoside, a new bitter triterpene glucoside from Ilex rotunda. Bull Chem Soc Jpn 62: 469-473, 1989.

29. Cui CZ, Wen XS, Cui M, Gao J, Sun B and Lou HX: Synthesis of solasodine glycoside derivatives and evaluation of their cytotoxic effects on human cancer cells. Drug Discov Ther 6: 9-17, 2012. 